\title{
RESENHA DA OBRA "DIREITO DIGITAL: DIREITO PRIVADO E INTERNET", COORDENADA POR GUILHERME MAGALHÃES MARTINS E JOÃO VICTOR ROZATTI LONGHI
}

José Luiz de Moura Faleiros Júnior ${ }^{1}$

Em 2014, ano em que foi promulgado o Marco Civil da Internet (Lei no 12.965), o Professor Guilherme Magalhães Martins, Promotor de Justiça titular da 5 - Rio de Janeiro e Professor adjunto de Direito Civil da Faculdade Nacional de Direito - UFRJ, lançou ao mercado a obra coletiva "Direito Privado e Internet", tendo como pedra fundamental o grupo de pesquisa Relações Privadas e Internet, por ele coordenado no âmbito do Programa de PósGraduação em Direito (Mestrado e Doutorado) da UERJ, com a participação dos pesquisadores João Victor Rozatti Longhi, Pedro Marcos Nunes Barbosa, Thaita Trevizan, Pedro Modenesi, Helen Lima Orleans e Vinícius Klein. Ainda, os estudos que se seguiram contaram com contributos de Fernanda Nunes Barbosa, Vitor Almeida, Gabriel Furtado, Antonio dos Reis Pereira da Silva Júnior e Paula Moura Francesconi Pereira, sacramentando-se, por fim, com textos de renomados autores convidados, a saber: Danilo Doneda, Allan Rocha de Souza, Sérgio Branco, Ana Paula BarbosaFohrmann, Carlos Affonso Pereira de Souza, Renato Porto, Cláudio Lins de Vasconcelos, Helder Galvão e Antonia Espíndola Longoni Klee.

Enorme foi o sucesso da obra e, nos cinco anos subsequentes ao seu lançamento, inúmeras transformações, especialmente nos panoramas jurídico-normativo e jurisprudencial, despertaram a inquietude destes e de outros pesquisadores de escol, dando azo ao projeto de uma segunda edição da bem-sucedida empreitada.

Formatou-se, então, a parceria do Professor Guilherme Martins com o Professor João Victor Rozatti Longhi para a coordenação do novo compêndio de estudos, tendo cada um dos autores revisado e atualizado seu respectivo texto, ao passo que novos convites foram feitos e se somaram

\footnotetext{
${ }^{1}$ Mestrando e Bacharel em Direito pela Faculdade de Direito da Universidade Federal de Uberlândia (UFU). Especialista em Direito Processual Civil, Direito Civil e Empresarial, Direito Digital e Compliance. Advogado. ORCID 0000000201922336. E-mail: juniorfaleiros@outlook.com
} 
ao já seleto rol de pesquisadores os seguintes estudiosos das relações entre o direito privado e a Internet: Ana Amélia Menna Barreto, Gabriel Oliveira de Aguiar Borges, José Luiz de Moura Faleiros Júnior, Juliano Madalena, Manuel David Masseno, Renato Opice Blum, Sophia Martini Vial, Têmis Limberger e Walter Aranha Capanema.

Revisitando o título da obra, os Professores Guilherme e João Victor optaram por inserir a expressão "Direito Digital", reenquadrando a vetusta denominação como subtítulo para denotar a expansão desta segunda edição, cujos novos textos reforçam o caráter denso e profícuo do trabalho, que permanece voltado às seguintes três grandes linhas: situações jurídicas existenciais na sociedade da informação, a proteção do consumidor na Internet e direitos autorais e tecnologia.

Na primeira parte, cujo foco é o estudo das situações jurídicas existenciais no contexto da hodierna sociedade da informação², o texto de Ana Paula Barbosa-Fohrmann e Antonio dos Reis Silva Júnior (p. 3-34) se dedica à investigação do discurso de ódio (hate speech), explorando farta casuística que dá sustentação às proposições apresentadas. Em sequência, Danilo Doneda (p. 3554) explicita suas considerações sobre a proteção de dados pessoais, elegendo razões para sua elevação ao patamar de direito fundamental - baliza essencial para a compreensão de arquétipos normativos recentíssimos, como o Regulamento Geral de Proteção de Dados europeu (GDPR, na sigla em inglês) e a Lei Geral de Proteção de Dados brasileira (Lei no 13.709/2018).

O pesquisador Gabriel Borges (p. 55-66), em estudo de caso, aborda o contraponto da liberdade de imprensa aos direitos à imagem, à intimidade e à privacidade na Internet. Segue-se o texto do Professor Guilherme Martins (p. 67-94), com densa abordagem acerca de um dos assuntos mais inquietantes da atualidade: o direito ao esquecimento. Ainda, denotando a variedade de temas na interdisciplinaridade almejada pela obra no campo do direito privado, Helen Cristina Orleans ( $p$. 95-122) se debruça sobre a desafiadora questão da infidelidade virtual.

Em considerações técnicas, o Professor João Victor Longhi (p. 123-154) estuda os fundamentos, os princípios, e o regime de responsabilidade civil adotados pelo Marco Civil da Internet. Por sua vez, José Faleiros Júnior (p. 155-182) apresenta um estudo de caso que se propõe a investigar a possibilidade de responsabilização do administrador de grupo da mídia social WhatsApp.

Juliano Madalena (p. 183-206) trabalha com nuances imperativas para que se caminhe adequadamente rumo à regulação das fronteiras da Internet; Pedro Marcos Nunes Barbosa (p. 207236) trabalha a intrincada questão dos signos distintivos na Internet, em contraposição à liberdade de

\footnotetext{
${ }^{2} \mathrm{O}$ conceito de sociedade da informação é comumente atribuído ao sociólogo espanhol Manuel Castells, mas é de se reconhecer que há certa controvérsia sobre as origens da expressão. Para alguns, o termo teria sido cunhado originalmente pelo norte-americano Fritz Machlup, em trabalhos dos anos 1960 e 1970; para outros, o termo teria surgido primeiramente nos escritos, também da década de 1960, dos japoneses Kisho Kurokawa, Tudao Umesao, Yujiro Hayashi e Yoneji Masuda (DUFF, Alistair A. Information Society Studies. Londres: Routledge, 2000, p. 3-4.). Não obstante, o aprimoramento conceitual trazido por Castells - e também pelo sociólogo holandês Jan van Dijk -, já na década de 1990, veio a indicar que as redes teriam o potencial de configurar uma nova morfologia social das sociedades, de modo que o conceito de ambos quanto à formatação de uma network society ("sociedade em rede") se diferencia do conceito de "sociedade da informação". Confirase: CASTELLS, Manuel. The rise of the network society. 2. ed. Oxford/West Sussex: Wiley-Blackwell, 2010 (The information age: economy, society, and culture, v. 1); VAN DIJK, Jan. The network society. 3. ed. Londres: Sage Publications, 2012.
} 
expressão. Renato Opice Blum (p. 237-252) faz apontamentos sobre a Internet das Coisas $^{3}$ elemento central da chamada web $4.0^{4}$ - e Têmis Limberger (p. 253-266) traz detalhado comparativo entre os regulamentos europeu e brasileiro de proteção de dados.

A primeira parte é concluída com os estudos de Thaita Trevizan (p. 267-282) acerca da tutela da imagem da pessoa humana à luz de estudos de caso da jurisprudência brasileira.

Percebe-se rica abordagem, com temas que - nesta primeira linha de estudos - apresenta ao leitor diversos pontos de contato do chamado "direito digital" com assuntos segmentados dos demais ramos do direito privado. Para além da questão regulatória, investiga-se cases, apresenta-se propostas para o equacionamento de disputas inovadoras e sensíveis do trato com a web e, com a densidade que marca o cariz investigativo de cada autor, incute-se no leitor rica experiência de cognição quanto aos desafios e às perspectivas de importantes temas cotidianos.

Avança-se, na segunda parte da obra, ao estudo da proteção do consumidor na Internet, tendo por balizas iniciais os textos de Allan Rocha de Souza (p. 283-302) sobre cultura, a revolução tecnológica e os direitos autorais, e de Ana Amélia Menna Barreto (p. 303-310) sobre o caminho da legalidade do bloqueio judicial do WhatsApp. Impostações preliminares de inquietantes situações que ilustram o matiz explorado na segunda parte.

O texto de Antonia Klee (p. 311-348) versa sobre o conceito de estabelecimento empresarial e remete a reflexões que se dedicam a trabalhar a proteção do consumidor nas contratações eletrônicas. Este tema se correlaciona aos textos de Pedro Modenesi (p. 433-498) e de Fernanda Nunes Barbosa (p. 349-374): o primeiro cuida dos aspectos doutrinário, legislativo e jurisprudencial dos contratos eletrônicos; o segundo, da proteção da privacidade do consumidor frente à oferta no mercado virtual.

Tem-se, ademais, texto do Professor Manuel David Masseno (p. 375-390), de Portugal, que fornece enriquecedora perspectiva para a proteção dos cidadãos-consumidores sob a ótica regulatória da União Europeia.

Rica casuística se apresenta em seguida, tendo, no texto de Paula Moura Francesconi Lemos Pereira (p. 391-432), interessante investigação sobre o uso da Internet na prestação de serviços médicos. Já Renato Porto (p. 499-510) se dedica ao estudo da influência midiática nas preferências de consumo do público infanto-juvenil. Por sua vez, Sophia Vial (p. 511-526) se volta ao estudo das moedas virtuais (e-money), delineando seus aspectos, funções e problemas.

Gabriel Furtado e Vitor Almeida (p. 543-576) analisam o comércio eletrônico coletivo e a tutela do consumidor nesse tipo de relação jurídica; Vinícius Klein (p. 527-542) faz um estudo de caso sobre contratações eletrônicas interempresariais; e, finalmente, Walter Capanema (p. 577-590) trata dos desafios da cooperação judicial das empresas que operam na Internet.

\footnotetext{
${ }^{3}$ Para melhor entendimento da expressão, sugere-se a seguinte leitura: GREENGARD, Samuel. The internet of things. Cambridge: The MIT Press, 2015, p. 188-189.

${ }^{4}$ A evolução histórica da Internet é representada pela doutrina em "eras" denominadas de web 1.0 a web 4.0, embora haja parcela da doutrina que já sinalize a existência de uma web 5.0 (PATEL, Karan. Incremental journey for world wide web: introduced with web 1.0 to recent web 5.0: a survey paper. International Journal of Advanced Research in Computer Science and Software Engineering, Jaunpur, v. 3, n. 10, p. 410-417, out. 2013, p. 416.)
} 
Novamente, percebe-se amplo rol de temas relacionados ao uso da Internet e, mais especificamente, à tutela das relações de consumo no plano virtual. E, sem deixar de observar a relevância do comércio eletrônico para a evolução econômica, tem-se em pauta uma série de indicações doutrinárias, jurisprudenciais e regulatórias que apontam para a necessidade de colmatação para a tutela de determinada casuística cuja repercussão - quase imediata, devido à pujança do avanço tecnológico -, tem o condão de colocar o ciberconsumidor ${ }^{5}$ em situação de risco (e vulnerabilidade).

$\mathrm{Na}$ terceira e derradeira parte da obra, analisa-se a relação entre os direitos autorais e a tecnologia, com quatro capítulos elaborados por estudiosos da matéria, em abordagens igualmente instigantes e propositivas.

Inicia-se com a pesquisa de Carlos Affonso Pereira de Souza (p. 591-616) sobre as transformações na criação e no licenciamento de obras intelectuais frente à proteção dos direitos de autor na sociedade da informação. Tema rico e extremamente atual, em um universo no qual a disponibilidade de amplo acesso à informação, na mesma medida em que democratiza o conhecimento, expõe a risco a proteção da propriedade intelectual.

Cláudio Lins de Vasconcelos (p. 617-638) faz intrigante abordagem acerca do chamado fair use, densamente estudado no direito norte-americano e, em relação às suas limitações decorrentes da presença da tecnologia, o autor propõe uma releitura da doutrina utilitarista, investigando seu cabimento no ordenamento brasileiro.

Helder Galvão (p. 639-654) analisa a questão do plágio e suas consequências jurídicas; por sua vez e, finalizando a obra, Sérgio Branco (p. 655-664) estuda didaticamente as chamadas licenças Creative Commons, indicando sua finalidade, os modos para seu licenciamento e o escopo de proteção desse modelo frente à proteção conferida pela legislação aos direitos autorais.

À guisa de conclusão, obtempera-se a relevância desta segunda edição da importante obra, na medida em que temas diversos e de grande impacto assolam os sistemas jurídicos na contemporaneidade, revelando preocupante movimentação tendente a prevenir que determinadas corporações ${ }^{6}$ se sobreponham ao próprio Estado. ${ }^{7} \mathrm{E}$, se a configuração de uma Lex Informatica ${ }^{8}$, como propunha Lessig na década de 1990, não parece ser viável, na medida em que tecnologia e inovação caminham em franco descompasso ${ }^{9}$ com o poder legiferante estatal, novas propostas

\footnotetext{
${ }^{5}$ A expressão é explorada, com profundidade, no capítulo de autoria de Pedro Modenesi, e, ainda, em: OLIVEIRA, Elsa Dias. A proteção dos consumidores nos contratos celebrados através da internet. Coimbra: Almedina, 2002, p. 57.

${ }^{6}$ Esta preocupação é manifestada, dentre outros, por Tim Wu, que aponta os riscos da elevação de poder dos chamados 'impérios da comunicação'. (WU, Tim. The master switch: the rise and fall of information empires. Nova York: Vintage, 2010.)

7 Confira-se: LLOYD, lan J. Information technology law. 6. ed. Oxford: Oxford University Press, 2011, p. 19; GOLDSMITH, Jack; WU, Tim. Who controls the Internet? Illusions of a borderless world. Oxford: Oxford University Press, 2006.

${ }^{8}$ LESSIG, Lawrence. Code, and other laws of cyberspace. Nova York: Basic Books, 1999, p. 43.

${ }^{9}$ Diz a doutrina: "The law and justice have lagged behind new technology in almost every period in history. This is understandable, as new technology must become established in society before legislation can be applied to it. Furthermore, the consequences of new technology are not always clear right away. That is why the legal answer usually has the character of a reaction or an adjustment of existing principles. In civil society, this character is enhanced by the principle of civil law, in which individuals initially act freely and the law subsequently makes corrections". (VAN DIJK, Jan. The network society..., p. 128)
} 
devem despertar no jurista o desejo pela investigação interdisciplinar deste chamado "direito digital" que é fruto das transformações sociais.

Se a vigilância "se insinua em estado líquido", como defendem Bauman e Lyon ${ }^{10}$, em clara alusão aos movimentos constantes dos fenômenos sociais e à carência de certezas e de vínculos duráveis, é sempre importante lembrarmo-nos de que "o outro lado da moeda, porém, é que organizações de todos os tipos nos conhecem simplesmente como sequências codificadas de números e letras". ${ }^{11}$

Nessa linha, sinalizações recentes como a edição da Medida Provisória no 869, de 2018 também contemplada nos estudos da obra -, no afã de suprir açodadamente uma lacuna importante da Lei Geral de Proteção de Dados, devem incutir no pesquisador do direito, destacadamente no que diz respeito às interações deste com a tecnologia, a curiosidade para ir além da rasa leitura que se pode fazer de legislações dessa natureza.

Assim, em um importante momento de convergência normativa internacional, em que diversos países editam marcos regulatórios para a proteção de dados e para a tutela de situações jurídicas na Internet, "Direito Digital: Direito Privado e Internet" surge como uma das mais ricas obras em Língua Portuguesa - dentro de seu recorte metodológico - acerca do tema, sendo leitura indispensável para qualquer operador do direito.

\section{REFERÊNCIAS}

BAUMAN, Zygmunt; LYON, David. Vigilância líquida. Tradução de Carlos Alberto Medeiros. Rio de Janeiro: Zahar, 2013.

CASTELLS, Manuel. The rise of the network society. 2. ed. Oxford/West Sussex: Wiley-Blackwell, 2010 (The information age: economy, society, and culture, v. 1)

DUFF, Alistair A. Information Society Studies. Londres: Routledge, 2000.

GOLDSMITH, Jack; WU, Tim. Who controls the Internet? Illusions of a borderless world. Oxford: Oxford University Press, 2006.

GREENGARD, Samuel. The internet of things. Cambridge: The MIT Press, 2015.

LESSIG, Lawrence. Code, and other laws of cyberspace. Nova York: Basic Books, 1999.

LLOYD, Ian J. Information technology law. 6. ed. Oxford: Oxford University Press, 2011.

LYON, David. The electronic eye: the rise of surveillance society. Minneapolis: University of Minnesota Press, 1994.

MARTINS, Guilherme Magalhães; LONGHI, João Victor Rozatti (Coord.). Direito digital: direito privado e internet. 2. ed. Indaiatuba: Foco, 2019.

${ }^{10}$ BAUMAN, Zygmunt; LYON, David. Vigilância líquida. Tradução de Carlos Alberto Medeiros. Rio de Janeiro: Zahar, 2013, p. 7.

${ }^{11}$ LYON, David. The electronic eye: the rise of surveillance society. Minneapolis: University of Minnesota Press, 1994, p. 3, tradução livre. 
VAN DIJK, Jan. The network society. 3. ed. Londres: Sage Publications, 2012.

OLIVEIRA, Elsa Dias. A proteção dos consumidores nos contratos celebrados através da internet. Coimbra: Almedina, 2002.

PATEL, Karan. Incremental journey for world wide web: introduced with web 1.0 to recent web 5.0: a survey paper. International Journal of Advanced Research in Computer Science and Software Engineering, Jaunpur, v. 3, n. 10, p. 410-417, out. 2013.

WU, Tim. The master switch: the rise and fall of information empires. Nova York: Vintage, 2010.

Como citar: FALEIROS JÚNIOR, José Luiz de Moura. Resenha da obra "Direito Digital: Direito Privado e Internet", coordenada por Guilherme Magalhães Martins e João Victor Rozatti Longhi. Revista IBERC, Minas Gerais, v. 2, n. 3, p. 1-6, set./dez. 2019. 